\title{
Rathke Pouch Cyst
}

National Cancer Institute

\section{Source}

National Cancer Institute. Rathke Pouch Cyst. NCI Thesaurus. Code C4977.

Rathke's pouch cysts are rarely symptomatic in the first two decades of life though they

may produce symptoms in the third and fourth decades of life. When the cysts do

present in the first decades of life, the symptoms are generally associated with diabetes insipidus or other conditions related to hypopituitarism. 